\title{
Anti-diabetic activity of chromium picolinate and biotin in rats with type 2 diabetes induced by high-fat diet and streptozotocin
}

\author{
Kazim Sahin $^{1 *}$, Mehmet Tuzcu ${ }^{2}$, Cemal Orhan ${ }^{1}$, Nurhan Sahin ${ }^{1}$, Osman Kucuk $^{3}$, \\ Ibrahim H. Ozercan ${ }^{4}$, Vijaya Juturu ${ }^{5}$ and James R. Komorowski ${ }^{5}$ \\ ${ }^{1}$ Department of Animal Nutrition, Faculty of Veterinary Science, Firat University, Elazig 23119, Turkey \\ ${ }^{2}$ Division of Biology, Faculty of Science, Firat University, Elazig 23119, Turkey \\ ${ }^{3}$ Department of Animal Nutrition and Nutritional Diseases, School of Veterinary Medicine, Erciyes University, \\ Kayseri 38039, Turkey \\ ${ }^{4}$ Department of Pathology, School of Medicine, Firat University, Elazig 23119, Turkey \\ ${ }^{5}$ Technical Services and Scientific Affairs, Nutrition 21 Inc., 4 Manhattanville Road, Purchase, NY 10577, USA \\ (Submitted 9 May 2012 - Final revision received 28 September 2012 - Accepted 29 September 2012 - First published online 5 December 2012)
}

\section{Abstract}

The objective of the present study was to evaluate anti-diabetic effects of chromium picolinate (CrPic) and biotin supplementations in type 2 diabetic rats. The type 2 diabetic rat model was induced by high-fat diet (HFD) and low-dose streptozotocin. The rats were divided into five groups as follows: (1) non-diabetic rats fed a regular diet; (2) diabetic rats fed a HFD; (3) diabetic rats fed a HFD and supplemented with CrPic $(80 \mu \mathrm{g} / \mathrm{kg}$ body weight (BW) per d); (4) diabetic rats fed a HFD and supplemented with biotin (300 $\mu \mathrm{g} / \mathrm{kg} \mathrm{BW}$ per d); (5) diabetic rats fed a HFD and supplemented with both CrPic and biotin. Circulating glucose, cortisol, total cholesterol, TAG, NEFA and malondialdehyde concentrations decreased $(P<0.05)$, but serum insulin concentrations increased $(P<0.05)$ in diabetic rats treated with biotin and $\mathrm{CrPic}$, particularly with a combination of the supplements. Feeding a HFD to diabetic rats decreased PPAR- $\gamma$ expression in adipose tissue and phosphorylated insulin receptor substrate 1 (p-IRS-1) expression of liver, kidney and muscle tissues, while the supplements increased $(P<0.001)$ PPAR- $\gamma$ and p-IRS-1 expressions in relevant tissues. Expression of NF- $\mathrm{kB}$ in the liver and kidney was greater in diabetic rats fed a HFD, as compared with rats fed a regular diet $(P<0 \cdot 01)$. The supplements decreased the expression of NF- $\mathrm{B}$ in diabetic rats $(P<0.05)$. Results of the present study revealed that supplementing CrPic and biotin alone or in a combination exerts anti-diabetic activities, probably through modulation of PPAR- $\gamma$, IRS-1 and NF- $\kappa$ B proteins.

Key words: Chromium: Biotin: Glucose: Insulin: PPAR- $\gamma$ : Phosphorylated insulin receptor substrate-1: NF-кB

Insulin resistance has a principal importance for the development and progression of diabetes as well as the metabolic syndrome, obesity, hypertension and $\mathrm{CVD}^{(1,2)} \mathrm{Cr}$ is considered an essential factor for optimal insulin activity and normal carbohydrate/lipid mechanism ${ }^{(3)}$. Biotin is an essential cofactor for a number of carboxylases that are important in carbohydrate and lipid metabolism, including acetyl-CoA carboxylase and methylcrotonyl-CoA carboxylase. Biotin stimulates the activity of glucokinase in the liver and improves pancreatic $\beta$ islet cell function to enhance insulin regulation ${ }^{(4)}$.

In vitro data have suggested that the combination of $10 \mathrm{ng} /$ $\mathrm{ml}$ chromium picolinate (CrPic) with $50 \mathrm{mg} / \mathrm{kg}$ biotin greatly enhances glucose uptake and glycogen production in a human skeletal muscle culture ${ }^{(5)}$. It was reported that CrPic, and the combination of CrPic and biotin, are capable of enhancing glucose disposal, lowering total cholesterol (TC) levels and increasing HDL-cholesterol levels in people with type 2 diabetes ${ }^{(6)}$. The combination of $\mathrm{CrPic}$ and biotin has also been reported to improve glycaemic control in overweight uncontrolled type 2 diabetes ${ }^{(7-9)}$. In human studies, CrPic and biotin reduced cholesterol levels ${ }^{(7)}$ and atherogenic index in plasma ${ }^{(10)}$. The mechanism by which $\mathrm{Cr}$ and biotin exert their anti-diabetic effects has not been studied in detail. PPAR- $\gamma$ and insulin receptor substrate 1 (IRS-1) are important biomarkers involved in insulin signalling and resistance as well as in inflammation ${ }^{(11,12)}$. NF- $\kappa \mathrm{B}$ is another biomarker involved in cellular responses to stimuli such as stress and free radicals ${ }^{(13)}$. Hua et al. ${ }^{(14)}$ stated that $\mathrm{Cr}$

Abbreviations: AIN, American Institute of Nutrition; BW, body weight; CISI, composite insulin sensitivity index; CrPic, chromium picolinate; HFD, high-fat diet; HOMA-IR, homeostasis model assessment-estimated insulin resistance; IRS-1, insulin receptor substrate 1; MDA, malondialdehyde; p-IRS-1, phosphorylated insulin receptor substrate 1; STZ, streptozotocin; TC, total cholesterol.

*Corresponding author: Dr K. Sahin, fax +90 42423881 73, email nsahinkm@yahoo.com 
down-regulates proteins such as IRS-1 involved in insulin resistance. Similarly, Sharma et al. ${ }^{(15)}$ found that improved glycaemic control is associated with increased PPAR- $\gamma$ and decreased NF- $\kappa \mathrm{B}$ protein expression in high-fat diet (HFD)streptozotocin (STZ)-induced type 2 diabetic rats. Therefore, the aim of the present study was to evaluate the anti-diabetic properties of CrPic and biotin and their action of mechanism by looking at the changes in biomarkers such as PPAR- $\gamma$, IRS-1 and NF-кB proteins, antioxidant status measured as malondialdehyde (MDA), metabolic parameters, as well as tissue histopathological changes in STZ-induced type 2 diabetic rats fed a HFD.

\section{Experimental methods}

\section{Animals and diets}

Male Sprague-Dawley rats were purchased from the Firat University Laboratory Animal Research Center (Elazig, Turkey). The animals were reared at the temperature of $22 \pm 2^{\circ} \mathrm{C}$, humidity of $55 \pm 5 \%$ and with a $12 \mathrm{~h}$ light $-12 \mathrm{~h}$ dark cycle. All animal procedures were approved by the Animal Experimentation Ethics Committee of Firat University (Elazig, Turkey). All procedures involving rats were conducted in strict compliance with the relevant laws, the Animal Welfare Act, Public Health Services Policy and guidelines established by the Institutional Animal Care and Use Committee of the Institute. Prior to the starting of the experiment, animals were assigned to either a regular diet (control; $12 \%$ of energy from fat) or a HFD ( $42 \%$ of energy from fat). The control diet and HFD were composed, according to the American Institute of Nutrition (AIN)-93 ${ }^{(16)}$ recommendations, of casein (20\%), soyabean oil (7\%), wheat starch $(53 \cdot 2 \%)$, sucrose (10\%), potato starch (5\%), L-cysteine $(0 \cdot 3 \%)$, vitamin mix AIN-93M (1\%) and mineral mix AIN-93M (3.5\%). The HFD ( $42 \%$ energy from fat) was obtained from the basal AIN-93 diet, by replacement of wheat starch with fat (tallow 15\% and soyabean oil 10\%). The chemical composition of experimental diets is shown in Table 1. For induction of type 2 diabetes (insulin resistance) and dyslipidaemia, the rats were fed the HFD for 2 weeks, fasted overnight at the last day and received a single injection of STZ (Sigma Chemical Company) intraperitoneal at the end of the $14 \mathrm{~d}$. STZ was dissolved in citrate buffer ( $\mathrm{pH} 4.5$ ) and injected at $40 \mathrm{mg} / \mathrm{kg}$ body weight (BW). A control group was given citrate buffer via intraperitoneal injection. The development of hyperglycaemia in rats was confirmed by measuring fasting serum glucose at $72 \mathrm{~h}$ after STZ injection. Rats with a fasting serum glucose level above $1400 \mathrm{mg} / \mathrm{l}$ were considered diabetic and were recruited to the study.

Table 1. Macronutrient composition of the control and high-fat diet (HFD) fed to rats

\begin{tabular}{lcc}
\hline & \multicolumn{2}{c}{ Diet composition (\% energy) } \\
\cline { 2 - 3 } & Control & HFD \\
\hline Protein & 28 & 20 \\
Carbohydrate & 60 & 38 \\
Fat & 12 & 42 \\
\hline
\end{tabular}

\section{Experimental design}

The rats weighing 200-220 g ( 8 weeks old, $n$ 75) were divided according to BW, which were similar, into five equal groups containing fifteen rats each. The groups were as follows: group 1: normoglycaemic rats, fed a regular diet (normal control) and injected with buffer solution as placebo; group 2: diabetic rats, fed a HFD without any supplementation (diabetic control); group 3: diabetic rats, fed a HFD supplemented with $\mathrm{CrPic}(80 \mu \mathrm{g} / \mathrm{kg}$ BW per $\mathrm{d})$; group 4: diabetic rats, fed a HFD supplemented with biotin $(300 \mu \mathrm{g} / \mathrm{kg}$ BW per d); group 5: diabetic rats, fed a HFD supplemented with both CrPic $(80 \mu \mathrm{g} / \mathrm{kg}$ BW per d) and biotin $(300 \mu \mathrm{g} / \mathrm{kg} \mathrm{BW}$ per $\mathrm{d}$ ). The CrPic and biotin supplements were dissolved in drinking-water and offered to rats. CrPic was supplemented to provide $8 \mu \mathrm{g} \mathrm{Cr} / \mathrm{d}$, which is an equivalent dose of $560 \mu \mathrm{g}$ $\mathrm{Cr}$ for a $70 \mathrm{~kg}$ adult human. Chromium picolinate and biotin were supplied by Nutrition 21, Inc. The study was carried out for 10 weeks. At the end of the experiment, all rats were killed by cervical dislocation. Blood samples were taken from rats in the morning, after overnight fasting, and the tissues from the pancreas, liver, kidney and muscles from the hind limb, including biceps femoris, gastrocnemius and soleus, were removed and processed for biochemical, Western blot and histopathological examination. Fat was trimmed off from the muscles. Feed intake and BW were measured twice weekly.

\section{Laboratory analyses}

Blood samples were centrifuged at $3000 \mathrm{~g}$ for $10 \mathrm{~min}$ and analysed for TC, TAG, insulin, cortisol, urea, creatinine and MDA. Estimation of insulin sensitivity from an oral glucose tolerance test, blood glucose and plasma insulin profiles were performed using the composite insulin sensitivity index (CISI) proposed by Matsuda \& De Fronzo ${ }^{(17)}$. Calculation of the index was made according the following equation:

$$
\mathrm{CISI}=10000 /((\mathrm{FPG} \times \mathrm{FPI}) \times(\mathrm{MG} \times \mathrm{MI}))^{1 / 2},
$$

where FPG and FPI are fasting plasma glucose and insulin concentrations, respectively, and MG and MI are the mean glucose and insulin concentrations, respectively, over the course of the oral glucose tolerance test.

At 1 week before surgical preparation, an oral glucose tolerance test was performed after $12-14 \mathrm{~h}$ of fasting on all animals. Blood glucose was measured in triplicate before (baseline) and 5, 15, 30, 45, 60, 90 and $120 \mathrm{~min}$ after the administration of $20 \%(\mathrm{w} / \mathrm{v})$ D-glucose solution ( $1 \mathrm{~g} / \mathrm{kg} \mathrm{BW})$ by oral administration. Blood was obtained from the tail vein and glucose was assessed using a glucometer (AccuChek Active; Roche Diagnostics).

A computer-solved model called the homeostasis model assessment-estimated insulin resistance (HOMA-IR) was calculated, as proposed by Muniyappa et al. ${ }^{(18)}$, using the following equation:

$$
\begin{aligned}
\text { HOMA-IR }= & (\text { fasting glucose }(\mathrm{mmol} / \mathrm{l}) \\
& \times \text { fasting insulin }(\mu \mathrm{IU} / \mathrm{ml})) / 22 \cdot 5 .
\end{aligned}
$$


Serum glucose concentrations were measured by using ACCU-Chek Active (Roche Diagnostics). Serum insulin levels were measured using the Rat Insulin Kit (Linco Research, Inc.) by ELISA (Elx-800; Bio-Tek Instruments, Inc.). Cortisol concentration was determined by ELISA using a commercially available kit, according to the manufacturer's protocol (Cortisol ELISA Kit no. 402710, assay range $0.05-5.0 \mathrm{ng} / \mathrm{ml}$; Neogen Corporation). Serum concentrations of TC and TAG were measured using the diagnostic kits (Sigma Diagnostics, Inc.). NEFA levels were measured by a diagnostic kit (Boehringer Mannheim, GmbH). Serum urea and creatinine concentrations and aspartate aminotransferase and alanine aminotransferase activities were measured using a biochemical analyser (Olympus AU-660, Olympus).

Lipid peroxidation was measured in terms of MDA formation, which is the major product of membrane lipid peroxidation, using a previously described method ${ }^{(19)}$ with slight modification. MDA concentration of kidney tissue was measured by HPLC (Shimadzu) using a Shimadzu UV-vis SPD-10 AVP detector and a C18-ODS-3, $5 \mu \mathrm{m}, 4.6 \times 250 \mathrm{~mm}$ column. The mobile phase was $30 \mathrm{~mm}-\mathrm{KH}_{2} \mathrm{PO}_{4}-$ methanol $(82 \cdot 5: 17 \cdot 5, \mathrm{v} / \mathrm{v}, \mathrm{pH} 3.6)$ and the flow rate was $1.2 \mathrm{ml} / \mathrm{min}$. Chromatograms were monitored at $250 \mathrm{~nm}$ and the injection volume was $20 \mu l$.

\section{Western blot}

Protein extraction was performed by homogenising the rat adipose, liver and kidney tissues in $1 \mathrm{ml}$ ice-cold hypotonic buffer A containing $10 \mathrm{~mm}$-HEPES (pH 7.8), $10 \mathrm{~mm}-\mathrm{KCl}$, $2 \mathrm{~mm}^{-\mathrm{MgCl}_{2}}, 1 \mathrm{~mm}$-dithiothreitol (DTT), $0.1 \mathrm{~mm}$-EDTA and $0 \cdot 1 \mathrm{~mm}$-phenylmethylsulfonyl fluoride. To the homogenate, $80 \mu \mathrm{l}$ of $10 \%$ Nonidet P-40 solution were added and the mixture was centrifuged for $2 \mathrm{~min}$ at $14000 \mathrm{~g}$. The precipitates, containing nuclei, were washed once with $500 \mu$ l of buffer A plus $40 \mu \mathrm{l}$ of $10 \%$ Nonidet P-40, centrifuged, resuspended in $200 \mu \mathrm{l}$ of buffer C (50 mm-HEPES (pH 7.8), $50 \mathrm{~mm}-\mathrm{KCl}$, $300 \mathrm{~mm}-\mathrm{NaCl}$, 0·1 mm-EDTA, $1 \mathrm{~mm}$-DTT, 0 1 mm-phenylmethylsulfonyl fluoride and $20 \%$ glycerol) and centrifuged for $5 \mathrm{~min}$ at $14800 \mathrm{~g}$. The supernatant proteins were collected for PPAR- $\gamma$, IRS-1 and NF- $\mathrm{BB}$. Equal amounts of protein $(50 \mu \mathrm{g})$ were electrophoresed and subsequently transferred to nitrocellulose membrane (Schleicher and Schuell, Inc.). The antibody against PPAR $\gamma$ phosphorylated tyrosine 612 IRS1 (Tyr162) and NF- $\mathrm{B}$ were purchased from Santa Cruz Biotechnology, Inc. Primary antibody was diluted (1:1000) in the same buffer containing $0.05 \%$ Tween-20. Protein loading was controlled using a monoclonal mouse antibody against $\beta$-actin antibody (A5316; Sigma). Bands were analysed densitometrically using an image analysis system (Image J; National Institutes of Health).

\section{Histopathological examination}

Following laparotomy, the tissues (liver, kidney and pancreas) of each rat were first examined grossly. The tissues were then removed for histological examination. Formaldehyde (10\%)-fixed pancreas, liver and kidney tissues were embedded in paraffin and serial sections $(3 \mathrm{~mm})$ were cut using a microtome. Each section was stained with haematoxylin and eosin for histological examination according to a standard procedure $^{(20)}$. The histological analyses were performed by the same person who was blinded to the group that each sample came from.

\section{Statistical analysis}

Sample size was calculated based on a power of $85 \%$ and a $P$ value of 0.05 . On the basis of this assumption, a sample size of fifteen per treatment was used. The data were analysed using the general linear model procedure of SAS (SAS Institute Inc.). Differences between groups were analysed using Fisher's multiple comparison test followed by Fisher's post hoc test for all groups.

\section{Results}

Effects of chromium picolinate and biotin on glucose, insulin, lipid profile and serotonergic properties

Feed intake and final BW were greater in diabetic rats fed either feeds supplemented with CrPic or biotin alone or as a combination $(P<0 \cdot 05)$. The effects of CrPic and biotin supplementations alone or in a combination on the serum glucose, insulin, cortisol and lipid profile of diabetic rats are presented in Table 2. After 10 weeks of feeding the HFD along with intraperitoneal injection of STZ, serum glucose, cortisol, TC, TAG and NEFA levels were elevated by 4.6-, 1·6-, 2.6-, 2.9- and 2.4-fold, respectively, in diabetic control rats, compared with the normal controls $(P=0 \cdot 0001)$. However, serum insulin and CISI values were reduced by $2 \cdot 1$ - and $3 \cdot 2$-fold in diabetic control rats, compared with rats fed a regular diet $(P=0 \cdot 0001)$. Diabetic rats fed a diet supplemented with CrPic or biotin had greater CISI, but lower HOMA-IR $(P<0.05)$. Either in combination or alone, CrPic and biotin decreased blood glucose, serum cortisol, TC, TAG and NEFA concentrations $(P=0.0001)$, but increased serum insulin and CISI concentrations in diabetic rats $(P=0 \cdot 0001)$. The effect of combination of $\mathrm{CrPic}$ and biotin was more pronounced on these parameters. In addition, except total TC concentration, CrPic was more effective compared with biotin on these parameters.

Diabetic rats had increased levels of alanine aminotransferase and aspartate aminotransferase and also urea and creatinine, compared with normal (non-diabetic) rats (Table 3). We found a 1.9-fold increase in serum urea, a $1 \cdot 7$-fold increase in creatinine, a $2 \cdot 1$-fold increase in alanine aminotransferase and a 1.9-fold increase in aspartate aminotransferase in HFD-STZ-treated diabetic control rats, compared with normal controls $(P=0 \cdot 0001)$. CrPic and biotin supplementations, alone or in combination, decreased serum urea, creatinine, aspartate aminotransferase and alanine aminotransferase levels in diabetic rats. The effect of the combination of CrPic and biotin was more pronounced on these parameters. Although not significant, CrPic was more effective on these parameters compared with biotin. 
Table 2. Effects of chromium picolinate ( $\mathrm{CrPic}$ ) and biotin supplementation on glucose, insulin and lipid profile in diabetic rats (Mean values with their standard errors of fifteen rats from each group)

\begin{tabular}{|c|c|c|c|c|c|c|c|c|c|c|}
\hline \multirow[b]{3}{*}{ Item } & \multicolumn{10}{|c|}{ Treatments } \\
\hline & \multicolumn{2}{|c|}{ Control } & \multicolumn{2}{|c|}{$\mathrm{HFD} / \mathrm{STZ}$} & \multicolumn{2}{|c|}{ HFD/STZ + CrPic } & \multicolumn{2}{|c|}{ HFD/STZ + biotin } & \multicolumn{2}{|c|}{$\underset{\text { biotin }}{\mathrm{HFD} / \mathrm{STZ}}+\mathrm{CrPic}+$} \\
\hline & Mean & SE & Mean & SE & Mean & SE & Mean & $\mathrm{SE}$ & Mean & SE \\
\hline Final body weight (g) & $320 \cdot 47^{a}$ & $5 \cdot 22$ & $216 \cdot 25^{c}$ & 3.51 & $240 \cdot 20^{b}$ & $3 \cdot 17$ & $235 \cdot 60^{b}$ & 7.57 & $248 \cdot 27^{b}$ & $6 \cdot 81$ \\
\hline Feed intake $(\mathrm{g} / \mathrm{d})$ & $29 \cdot 08^{a}$ & 0.53 & $19.53^{\mathrm{C}}$ & 0.32 & $21.82^{b}$ & 0.29 & $20 \cdot 46^{\mathrm{b}, \mathrm{c}}$ & 0.73 & $22.55^{\mathrm{b}}$ & 0.61 \\
\hline Glucose (mg/l) & $1030^{\mathrm{e}}$ & $5 \cdot 7$ & $4690^{\mathrm{a}}$ & $27 \cdot 8$ & $2870^{c}$ & $8 \cdot 6$ & $4190^{\mathrm{b}}$ & $8 \cdot 7$ & $2441 \cdot 8^{d}$ & $10 \cdot 2$ \\
\hline Insulin (mg/l) & $486.0^{\mathrm{a}}$ & $3 \cdot 2$ & $232 \cdot 1^{\mathrm{e}}$ & 1.6 & $258 \cdot 7^{\mathrm{c}}$ & $1 \cdot 7$ & $246.0^{d}$ & 1.9 & $272 \cdot 1^{\mathrm{b}}$ & $1 \cdot 7$ \\
\hline $\mathrm{CISI}$ & $2 \cdot 68^{a}$ & 0.01 & $0.83^{e}$ & 0.01 & $1 \cdot 28^{c}$ & 0.01 & $1 \cdot 21^{d}$ & 0.01 & $1.54^{\mathrm{b}}$ & 0.01 \\
\hline HOMA-IR & $12 \cdot 35^{\mathrm{e}}$ & 0.11 & $26 \cdot 85^{\mathrm{a}}$ & 0.26 & $18 \cdot 31^{c}$ & 0.11 & $25 \cdot 43^{\mathrm{b}}$ & 0.23 & $16 \cdot 39^{d}$ & 0.14 \\
\hline Cortisol (mg/l) & $82 \cdot 1^{d}$ & 0.6 & $134 \cdot 3^{a}$ & 1.3 & $115 \cdot 3^{\mathrm{C}}$ & 1.3 & $121 \cdot 1^{\mathrm{b}}$ & 1.2 & $113 \cdot 5^{\mathrm{c}}$ & 1.3 \\
\hline $\mathrm{TC}(\mathrm{mg} / \mathrm{l})$ & $950.7^{e}$ & 8.0 & $2503 \cdot 8^{a}$ & 12.5 & $2280.0^{\mathrm{b}}$ & $7 \cdot 0$ & $2230.0^{c}$ & $7 \cdot 0$ & $2013 \cdot 6^{d}$ & $5 \cdot 4$ \\
\hline $\mathrm{TAG}(\mathrm{mg} / \mathrm{l})$ & $1350 \cdot 7^{d}$ & $18 \cdot 1$ & $3902 \cdot 5^{\mathrm{a}}$ & 27.5 & $3659.0^{\mathrm{b}}$ & $18 \cdot 3$ & $3700.0^{\mathrm{b}}$ & $13 \cdot 4$ & $3429 \cdot 1^{c}$ & 13.8 \\
\hline $\operatorname{NEFA}(\mathrm{mmol} / \mathrm{l})$ & $1.60^{\mathrm{d}}$ & 0.06 & $3.90^{\mathrm{a}}$ & 0.08 & $3.31^{b}$ & 0.08 & $3.49^{b}$ & 0.08 & $2.82^{\mathrm{c}}$ & 0.05 \\
\hline
\end{tabular}

HFD, high-fat diet; STZ, streptozotocin; CISI, composite of insulin sensitivity index; HOMA-IR, homeostasis model assessment-estimated insulin resistance; TC, total cholesterol.

a,b,c,d,e Mean values within a row with unlike superscript letters were significantly different $(P<0.05)$.

Serum, liver and kidney MDA levels were increased upon feeding the HFD in diabetic rats $(P=0 \cdot 0001$; Table 4). The effect of the combination of CrPic and biotin was more pronounced on these parameters. In addition, except for liver MDA levels, CrPic was more effective compared with biotin on these parameters.

\section{Effects of chromium picolinate and biotin on protein expression of PPAR- $\gamma$, insulin receptor substrate 1} and $N F-\kappa B$ in tissues of diabetic rats

As shown in Fig. 1, feeding a HFD to rats decreased PPAR- $\gamma$ expression in adipose tissue, while CrPic or biotin, each alone or their combination, increased $(P<0.001)$ PPAR- $\gamma$ expression in this tissue. The level of phosphorylated IRS-1 (p-IRS-1) (Tyr162) in liver, kidney and muscle tissues of diabetic control rats was reduced to $52.33 \%$ of that in normal control rats $(P<0.05)$ (Fig. 2). CrPic or biotin alone similarly increased the p-IRS-1 expression, but the magnitude of the increase was more in case of the combination of supplements. Expression of NF- $\mathrm{KB}$ in the liver and kidney tissues was greater in rats fed a HFD, as compared with rats fed a regular diet $(P<0.01)$ (Fig. 3). Supplementing CrPic or biotin, or a combination of them, resulted in a decrease in expression of $\mathrm{NF}-\mathrm{\kappa B}$ in diabetic rats $(P<0 \cdot 05)$

\section{Effects of chromium picolinate and biotin on histopathology of tissues}

Histopathological evaluation of various liver, kidney and pancreatic segments of the rats fed a regular diet and rats fed a HFD are presented in Fig. 4.

Liver. Normal histology and normal hepatocellular architecture with a normal central vein were observed in nondiabetic rats. Hepatocytes having pink eosinophillic cytoplasm without any inclusions and with mostly central single nuclei were observed in non-diabetic rats. These cells had welldefined cell borders, polygonal in shape and arranged in sheets. Liver sinusoids were not dilated. There were no areas of haemorrhage or fibrosis. In diabetic rats, hepatocytes had vacuolated eosinophillic cytoplasm with a ground glass appearance and with fatty inclusions. The nuclei were enlarged, displaced and vacuolated. There were several pycnotic nuclei, suggesting significant hepatocellular

Table 3. Effects of chromium picolinate ( $\mathrm{CrPic}$ ) and biotin supplementation on urea, creatinine, aspartate aminotransferase (AST) and alanine aminotransferase (ALT) levels in diabetic rats

(Mean values with their standard errors of fifteen rats from each group)

\begin{tabular}{|c|c|c|c|c|c|c|c|c|c|c|}
\hline \multirow[b]{3}{*}{ Item } & \multicolumn{10}{|c|}{ Treatments } \\
\hline & \multicolumn{2}{|c|}{ Control } & \multicolumn{2}{|c|}{ HFD/STZ } & \multicolumn{2}{|c|}{$\begin{array}{l}\mathrm{HFD} / \mathrm{STZ}+ \\
\text { CrPic }\end{array}$} & \multicolumn{2}{|c|}{$\underset{\text { biotin }}{\mathrm{HFD} / \mathrm{STZ}}+$} & \multicolumn{2}{|c|}{$\begin{array}{l}\mathrm{HFD} / \mathrm{STZ}+ \\
\mathrm{CrPic}+\text { biotin }\end{array}$} \\
\hline & Mean & $\overline{\mathrm{SE}}$ & Mean & $\overline{S E}$ & Mean & $\overline{\mathrm{SE}}$ & Mean & $\overline{\mathrm{SE}}$ & Mean & $\overline{\mathrm{SE}}$ \\
\hline Urea $(\mathrm{mmol} / \mathrm{l})$ & $12 \cdot 16^{\mathrm{d}}$ & 0.19 & $23 \cdot 29^{\mathrm{a}}$ & 0.27 & $17.53^{b}$ & 0.22 & $17 \cdot 89^{\mathrm{b}}$ & 0.23 & $15 \cdot 80^{c}$ & 0.28 \\
\hline Creatinine $(\mu \mathrm{mol} / \mathrm{l})$ & $36 \cdot 24^{d}$ & 0.86 & $61 \cdot 88^{\mathrm{a}}$ & 0.79 & $49 \cdot 50^{\mathrm{b}}$ & 0.83 & $51 \cdot 27^{\mathrm{b}}$ & 0.98 & $45 \cdot 08^{\mathrm{C}}$ & 0.78 \\
\hline AST (U/I) & $115 \cdot 27^{d}$ & 1.73 & $241 \cdot 25^{\mathrm{a}}$ & $2 \cdot 34$ & $189 \cdot 00^{b}$ & $2 \cdot 86$ & $193 \cdot 00^{\mathrm{b}}$ & 1.51 & $171 \cdot 00^{c}$ & 1.41 \\
\hline ALT (U/I) & $80.00^{d}$ & 0.87 & $150 \cdot 00^{a}$ & 1.49 & $131.00^{\mathrm{b}}$ & 1.13 & $135.00^{\mathrm{b}}$ & 1.38 & $125.00^{C}$ & 1.21 \\
\hline
\end{tabular}

HFD, high-fat diet; STZ, streptozotocin.

$\mathrm{a}, \mathrm{b}, \mathrm{c}, \mathrm{d}$ Mean values within a row with unlike superscript letters were significantly different $(P<0.05)$. 
Table 4. Effects of chromium picolinate (CrPic) and biotin supplementation on serum, liver and kidney malondialdehyde (MDA) concentrations in diabetic rats

(Mean values with their standard errors of fifteen rats from each group)

\begin{tabular}{|c|c|c|c|c|c|c|c|c|c|c|}
\hline \multirow[b]{3}{*}{ Item } & \multicolumn{10}{|c|}{ Treatments } \\
\hline & \multicolumn{2}{|c|}{ Control } & \multicolumn{2}{|c|}{ HFD/STZ } & \multicolumn{2}{|c|}{$\begin{array}{l}\mathrm{HFD} / \mathrm{STZ}+ \\
\quad \text { CrPic }\end{array}$} & \multicolumn{2}{|c|}{$\begin{array}{l}\mathrm{HFD} / \mathrm{STZ}+ \\
\text { biotin }\end{array}$} & \multicolumn{2}{|c|}{$\begin{array}{l}\mathrm{HFD} / \mathrm{STZ}+ \\
\text { CrPic + biotin }\end{array}$} \\
\hline & Mean & SE & Mean & SE & Mean & SE & Mean & SE & Mean & SE \\
\hline Serum MDA (nmol/ml) & $0.93^{e}$ & 0.008 & $3.82^{a}$ & 0.006 & $2 \cdot 78^{\mathrm{c}}$ & 0.006 & $2 \cdot 90^{\mathrm{b}}$ & 0.004 & $2 \cdot 70^{d}$ & 0.04 \\
\hline Liver MDA (nmol/ml) & $6 \cdot 11^{d}$ & 0.069 & $11 \cdot 18^{a}$ & 0.073 & $10 \cdot 80^{\mathrm{b}}$ & 0.065 & $9 \cdot 47^{\mathrm{C}}$ & 0.060 & $9 \cdot 39^{c}$ & 0.058 \\
\hline Kidney MDA (nmol/ml) & $3.65^{\mathrm{e}}$ & 0.650 & $8 \cdot 16^{\mathrm{a}}$ & 0.960 & $6 \cdot 89^{\mathrm{c}}$ & 0.780 & $7 \cdot 22^{b}$ & 0.362 & $5 \cdot 12^{d}$ & 0.824 \\
\hline
\end{tabular}

HFD, high-fat diet; STZ, streptozotocin

$\mathrm{a}, \mathrm{b}, \mathrm{c}, \mathrm{d}, \mathrm{e}$ Mean values within a row with unlike superscript letters were significantly different $(P<0.05)$.

degeneration. The cells were irregular in size, shape and orientation. Moderate macrovesicular fatty degeneration of liver with dilated sinusoids was observed. In CrPic or biotin, or the combination of supplements groups, lobules with normal hepatocellular architecture with a normal central vein were observed. Hepatocytes had pink eosinophillic cytoplasm without any inclusions and with mostly central single nuclei. These cells had well-defined cell borders, polygonal in shape and arranged in sheets. Liver sinusoids were not dilated. There were no areas of haemorrhage or fibrosis.

Kidney. In diabetic (fat-fed/STZ) rats, renal parenchyma showing significant vacuolar degeneration of renal tubular cells with displaced pycnotic nuclei was observed. Renal glomeruli showing mesangial hyperplasia and eosinophillic fibrinous deposits were observed. Diffuse infiltration of monomorphonuclear cells in renal parenchyma and the glomeruli was observed. Tubulointerstitial nephropathy with significant mesangial proliferation in glomeruli was observed. In CrPic and biotin treatment or in combination groups, normal renal tubular architecture and normal glomeruli were observed.

Pancreas. Non-diabetic rats had normal exocrine pancreatic acinar architecture and pancreatic islets, showing predominantly insulin-producing $\beta$-cells with granular basophilic cytoplasm and few eosinophillic glucagon-producing $\alpha$-cells.
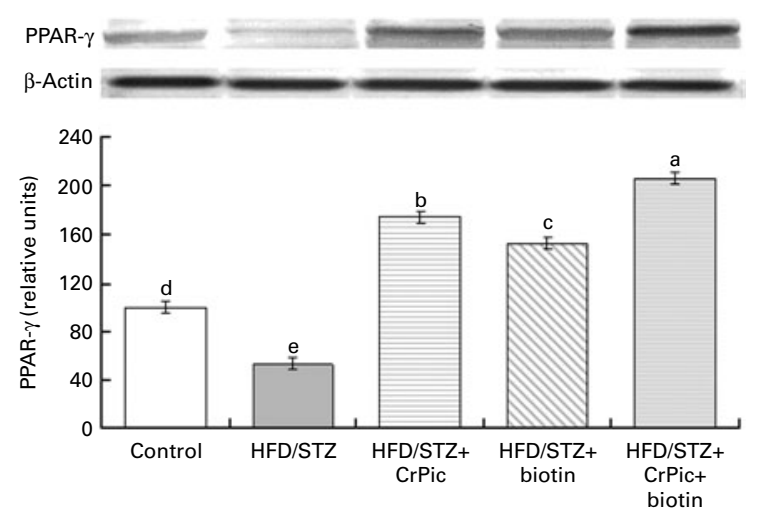

Fig. 1. Effects of diabetes and chromium picolinate (CrPic) and biotin supplementation on PPARy expression in adipose tissue. Data are expressed as a ratio of the normal control value (set to $100 \%$ ). Blots were repeated at least three times $(n 3)$ and a representative blot is shown. Actin was included to ensure equal protein loading. Values are means, with their standard errors represented by vertical bars. ${ }^{a, b, c, d, e}$ Mean values with unlike letters were significantly different $(P<0.05$; Fisher's multiple comparison test). HFD, high-fat diet; STZ, streptozotocin.
In diabetic rats, pancreatic islets showed a relatively decreased population of insulin-producing $\beta$-cells with granular basophilic cytoplasm and several eosinophillic glucagon-producing $\alpha$-cells. There were areas of eosinophillic amorphous deposits within islets, suggesting cellular necrosis. Degenerative changes and islet cell necrosis in endocrine pancreas were observed. In supplemental groups (CrPic or biotin or a combination of them), normal exocrine pancreatic acinar architecture was observed.

\section{Discussion}

Diabetes is the most common metabolic disorder worldwide and is associated with profound alterations in glucose, insulin, cortisol, lipid and lipoprotein profiles ${ }^{(15,21,22)}$. In the present study, Cr and biotin supplementation, each alone or together, ameliorated insulin resistance, dyslipidaemia, hepatic and renal damage and pathological changes in liver, kidney and pancreas tissues in HFD-STZ-induced type 2 diabetic rats. Similar to results of the present investigation, a number of studies have indicated that $\mathrm{CrPic}$ and biotin administration, each alone or together, can act as anti-diabetic agents and decrease TC concentration ${ }^{(7,8,23)}$. Previous studies have also demonstrated that animals, when fed a HFD and injected with a low dose of STZ, display many characteristics of insulin resistance, including hyperglycaemia and hyperinsulinaemia, decreased hyperlipidaemia, liver and kidney damage ${ }^{(15,22,23)}$. The effect of a combination of CrPic and biotin in the present study was more pronounced for all metabolic parameters, as well as MDA, and CrPic was more effective compared with biotin on most of the parameters in diabetic rats. Similarly, previous studies indicated that a combination of $\mathrm{CrPic}$ and biotin worked synergistically to produce positive effects on insulin function, blood glucose and lipid metabolism in people with type 2 diabetes $^{(7,8,10)}$ and in an animal model of insulin-resistant obesity ${ }^{(5)}$. In accordance with other metabolic parameters of the present study, diabetic rats fed a diet supplemented with CrPic or biotin had a greater CISI but lower HOMA-IR. Feed intake and final BW of diabetic rats were paralleled in a way that greater feed intake resulted in a greater final BW.

The present study provided evidence of a specific association between a combination of CrPic and biotin supplementation and MDA in diabetic rats. Lower levels of MDA were observed 

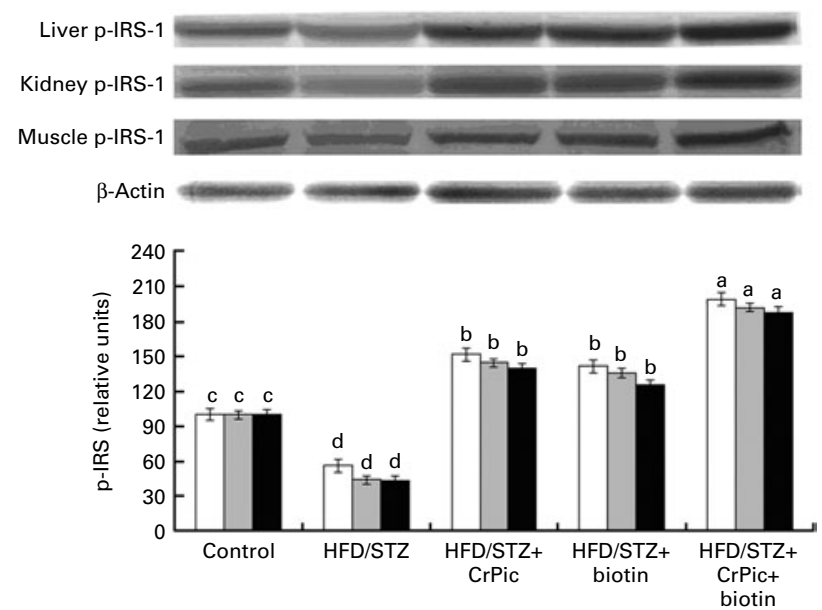

Fig. 2. Effects of diabetes and chromium picolinate (CrPic) and biotin supplementation on phosphorylation of insulin receptor substrate (p-IRS)-1)

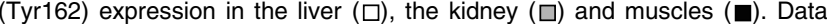
are expressed as a ratio of the normal control value (set to $100 \%$ ). Blots were repeated at least three times $(n 3)$ and a representative blot is shown. Actin was included to ensure equal protein loading. Values are means, with their standard errors represented by vertical bars. ${ }^{\text {a,b,c,d }}$ Mean values with unlike letters were significantly different $(P<0.05$; Fisher's multiple comparison test). HFD, high-fat diet; STZ, streptozotocin.

in diabetic animals receiving $\mathrm{CrPic}$ and biotin supplementation. Cr, through playing an important role against insulin resistance, is postulated to augment the antioxidant defence system $^{(23)}$. A decrease in hepatic thiobarbituric acid reactive substances formation by supplementation of $\mathrm{CrPic}$ and chromium nicotinate has been reported in rats ${ }^{(24)}$. It was also found that chromium dinicocysteinate supplementation in rats significantly lowered C-reactive protein and lipid peroxidation and increased vitamin $\mathrm{C}$ and adiponectin levels ${ }^{(25)}$. Oxidative stress associated with overproduction of reactive oxygen species plays an important role in the development of diabetic complications, including diabetic nephropathy ${ }^{(26)}$.

PPAR is a member of a nuclear receptor superfamily. Due to its role in decreasing insulin resistance and inflammation, PPAR has been involved in the pathology of numerous diseases, including obesity, diabetes and atherosclerosis ${ }^{(15,27)}$. PPAR exist as three different subtypes in mammals, namely, PPAR $\alpha$, PPAR $\delta$ and PPAR $\gamma$. PPAR $\gamma$, widely expressed in adipose tissue, is the central regulator of insulin and glucose metabolism, leading to improved insulin sensitivity in type 2 diabetic patients as well as in diabetic rodent models ${ }^{(21)}$. Several studies have shown that PPAR- $\gamma$ agonists may be effective in reducing renal cortical lipid content and proteinuria by a mechanism independent of their hypoglycaemic property ${ }^{(28-30)}$. In the present study, CrPic and biotin, as well as their combination, regulated (increased) PPAR- $\gamma$ expression in adipose tissue, which may exert a potent insulin-sensitising effect and may improve insulin resistance in a rat model of type 2 diabetes mellitus. It has been demonstrated that a combination of lipoic acid, acetyl-L-carnitine, nicotinamide and biotin treatment significantly increased mRNA levels of PPAR- $\gamma$ in skeletal muscles of type 2 diabetic Goto-Kakizaki rats $^{(31)}$. Additionally, it has been reported that anti-lipidaemic and anti-inflammatory effects of drugs, such as Semecarpus anacardium nut milk extract treatment, increase the mRNA expression of PPAR- $\gamma^{(32)}$.

Studies in diabetic mice and rat models have found that supplementing CrPic and biotin increases insulin sensitivity $^{(23,33,34)}$. IRS-1 appears to play a central role in the insulin-stimulated signal transduction pathway and links the insulin receptor to its final biological actions via a series of intermediate effectors ${ }^{(35)}$. Studies have shown that differential regulation of IRS-1 in the liver and muscle tissues were observed in diabetic animals, which may produce differential alterations in insulin signalling in these two tissues and contribute to hepatic and muscular insulin resistance ${ }^{(12)}$. In the present study, decreases of IRS-1 expression in the liver, kidney and muscle tissues were observed in diabetic rats. These results suggest that the favourable impact of $\mathrm{CrPic}$ and biotin on hyperlipidaemia was probably associated with the improvement of insulin signal transduction in target tissues, which plays an important role in regulating glucose metabolism. Consistent with the present findings, Jain et $a l .{ }^{(25)}$ reported that chromium dinicocysteinate supplementation increased IRS-1 activation in the liver tissues of type 2 diabetic rats. Similarly, Wang et al. ${ }^{(36)}$ reported that obese rats treated with CrPic had significantly improved glucose disposal rates and demonstrated a significant increase in insulinstimulated p-IRS-1 and phosphatidylinositol-3 kinase activity in skeletal muscles compared with obese controls.

$\mathrm{NF}-\kappa \mathrm{B}$, one of the transcription factors, is responsible for controlling DNA transcription and is involved in cellular responses to a number of stimuli including free radicals ${ }^{(13)}$. Hyperglycaemia-induced reactive oxygen species and advanced glycated end products stimulate the signal transduction pathway for the activation of the transcription factor $\mathrm{NF}-\kappa \mathrm{B}^{(37)}$. The results of the present study also showed that CrPic and biotin, as well as their combination, decreased $\mathrm{NF}-\kappa \mathrm{B}$ level in the liver and kidney tissues of the rats with

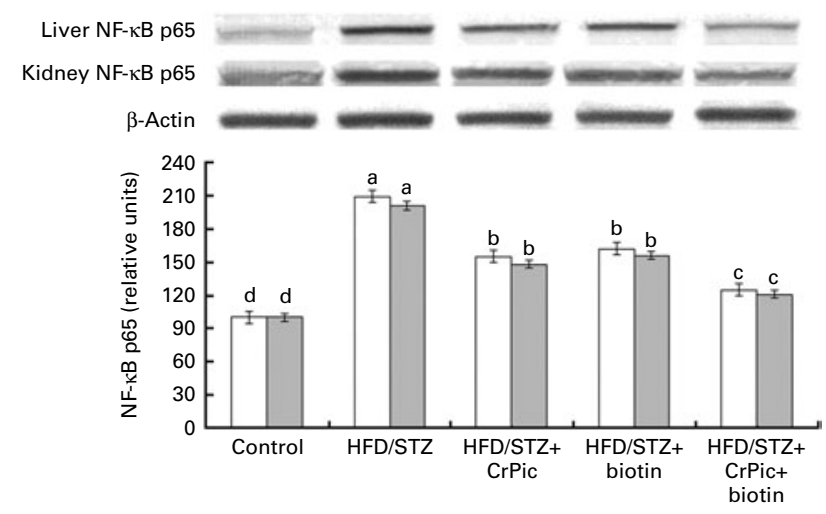

Fig. 3. Effects of diabetes and chromium picolinate (CrPic) and biotin supplementation on NF-kB p65 expression in the liver $(\square)$ and the kidney (口). Data are expressed as a ratio of the normal control value (set to $100 \%$ ). Blots were repeated at least three times $(n 3)$ and a representative blot is shown. Actin was included to ensure equal protein loading. Values are means, with their standard errors represented by vertical bars. ${ }^{a, b, c, d}$ Mean values with unlike letters were significantly different $(P<0.05$; Fisher's multiple comparison test). HFD, high-fat diet; STZ, streptozotocin. 


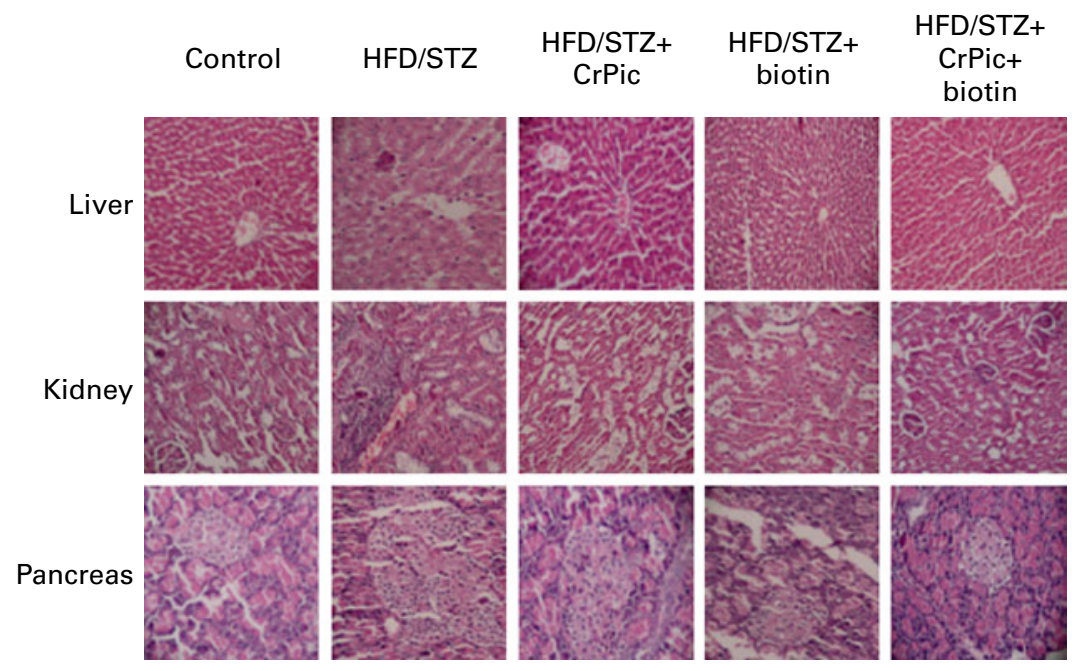

Fig. 4. Effects of chromium picolinate (CrPic) and biotin supplementation on histopathological changes in liver, kidney and pancreatic tissues (stained with haematoxylin and eosin dye). Control: liver, kidney and pancreas tissue sections of rats fed a regular diet; high-fat diet (HFD)/streptozotocin (STZ): liver, kidney and pancreas tissue sections from the diabetic rats; HFD/STZ + CrPic: liver, kidney and pancreas tissue sections from diabetic rats supplemented with CrPic; HFD/STZ + biotin: liver, kidney and pancreas tissue sections from diabetic rats supplemented with biotin; HFD/STZ + CrPic + biotin: liver, kidney and pancreas tissue sections from diabetic rats supplemented with both $\mathrm{CrPic}$ and biotin. (A colour version of this figure can be found online at http://www.journals.cambridge.org/bjn).

HFD/STZ-induced diabetes. In parallel with the results of the present study, Kuhad et al. ${ }^{(38)}$ reported that NF-kB p65 subunit was significantly elevated in the kidneys of diabetic animals. Results of the present investigation are also consistent with previous studies reporting that the levels of NF-кB p65 in the kidneys were distinctly increased in a lower-dose STZ-induced diabetic rat model fed a HFD ${ }^{(39)}$. Tuzcu et al. ${ }^{(40)}$ also reported a decrease in liver NF- $\mathrm{KB}$ expression by supplementation of $\mathrm{CrPic}$ in rats fed a HFD. In addition, Jain et $a l .{ }^{(25)}$ found that chromium dinicocysteinate is a potent hypoglycaemic compound, with anti-inflammatory activity apparently mediated by elevated blood vitamin $\mathrm{C}$ and inhibition of NF-кB, Akt and GLUT-2 in the livers of type 2 diabetic rats. It can be postulated from the present investigation and results from the reported studies ${ }^{(25,39,40)}$ that amelioration of insulin resistance by $\mathrm{CrPic}$ and biotin might be directly or indirectly associated with modulation of NF- $\mathrm{KB}$ expression.

Data found in the present study also provided evidence that HFD-STZ treatment produces liver, kidney and pancreatic lesions in rats because of associated hyperinsulinaemia, hyperlipidaemia, hyperglycaemia and increased oxidative stress. However, CrPic and biotin alone or in combination prevented these pathological alterations due to their insulin-sensitising, anti-inflammatory, anti-dyslipidaemia and antioxidant activity. Animal studies strongly support the hypoglycaemic action of biotin and $\mathrm{CrPic}$ via improved glucose tolerance and decreased insulin resistance ${ }^{(23,34,41)}$.

The present study clearly showed that supplementing CrPic or biotin alone or in a combination exerts antioxidant, antihyperlipidaemic, anti-inflammatory and anti-hyperglycaemic effects, and increases the level of insulin in diabetic rats. These changes may be related to the elevation of the levels of PPAR- $\gamma$ and P-IRS- 1 in the liver, kidney and muscle tissues. The combination of CrPic and biotin in the present study was more effective for all metabolic parameters, as well as on
MDA, PPAR- $\gamma$, p-IRS-1 and NF-кB. CrPic supplementation was more effective, compared with biotin supplementation, on most of the parameters in diabetic rats. Therefore, a nutritional supplement containing CrPic and biotin is highly recommended for individuals having metabolic diseases, diabetes in particular. CrPic and biotin supplementations could exert their health-benefiting roles in regulating diabetes via activation of PPAR- $\gamma$, IRS- 1 and NF- $\mathrm{BB}$ proteins and its associated complications in the tissues. The present doses of CrPic and biotin provided positive results, but dose-dependent responses could also be investigated as a further study.

\section{Acknowledgements}

The present study was supported by Nutrition 21 . The authors' responsibilities were as follows: C. O., M. T. and I. H. O. participated in data collection and laboratory analyses and assisted in every aspect of the study; N. S. participated in the study design, data collection, laboratory analyses and wrote the first draft of the manuscript; V. J. and J. R. K. participated in study design, interpretation and preparation of the manuscript; K. S. and O. K. participated in the organisation of the study and data interpretation and preparation of the manuscript. All authors read and approved the final manuscript. None of the authors has any conflicts of interest.

\section{References}

1. Kohli P \& Greenland P (2006) Role of the metabolic syndrome in risk assessment for coronary heart disease. JAMA 295, 819-821.

2. Cortez-Dias N, Martins S \& Fiuza M (2007) Metabolic syndrome: an evolving concept. Rev Port Cardiol 26, 1409-1421.

3. Anderson RA, Cheng N, Bryden NA, et al. (1997) Elevated intakes of supplemental chromium improve glucose and 
insulin variables in individuals with type 2 diabetes. Diabetes 46, 1786-1791.

4. Fernandez-Mejia C (2005) Pharmacological effects of biotin. J Nutr Biochem 16, 424-427.

5. Wang ZQ, Zhang XH, Baldor LC, et al. (2000) Chromium picolinate enhances insulin sensitivity in an animal model for the metabolic syndrome: the obese, insulin resistant JCR:LA-corpulent rat (abstract). Diabetes 49, A71.

6. Komorowski JR, Greenberg D, Maki KC, et al. (2001) Chromium picolinate with biotin attenuates elevation in blood glucose levels in people with type two diabetes ingesting medium carbohydrate nutritional beverages (abstract). $J \mathrm{Am}$ Coll Nutr 20, A73.

7. Albarracin CA, Fuqua BC, Evans JL, et al. (2008) Chromium picolinate and biotin combination improves glucose metabolism in treated, uncontrolled overweight to obese patients with type 2 diabetes. Diabetes Metab Res Rev 24, $41-51$.

8. Singer GM \& Geohas J (2006) The effect of chromium picolinate and biotin supplementation on glycemic control in poorly controlled patients with type 2 diabetes mellitus: a placebo-controlled, double-blinded, randomized trial. Diabetes Technol Ther 8, 636-643.

9. Juturu V, Ruelle A, Hudson MS, et al. (2006) Improved glycemic control after diabetes education and chromium picolinate/biotin supplementation in type 2 diabetes: results from patients experience pilot program. Trace Elem Electrolytes 23, 66-72.

10. Geohas J, Daly A, Juturu V, et al. (2007) Chromium picolinate and biotin combination reduces atherogenic index of plasma in patients with type 2 diabetes mellitus: a placebocontrolled, double-blinded, randomized clinical trial. Am J Med Sci 333, 145-153.

11. Hallakou S, Doaré L, Foufelle F, et al. (1997) Pioglitazone induces in vivo adipocyte differentiation in the obese Zucker fa/fa rat. Diabetes 46, 1393-1399.

12. Saad MJ, Araki E, Miralpeix M, et al. (1992) Regulation of insulin receptor substrate-1 in liver and muscle of animal models of insulin resistance. J Clin Invest 90, 1839-1849.

13. Gilmore JL, King BW, Harris C, et al. (2006) Synthesis and structure-activity relationship of a novel, achiral series of TNF-alpha converting enzyme inhibitors. Bioorg Med Chem Lett 16, 2699-2704.

14. Hua Y, Clark S, Ren J, et al. (2012) Molecular mechanisms of chromium in alleviating insulin resistance. $J$ Nutr Biochem 23, 313-319.

15. Sharma AK, Bharti S, Ojha S, et al. (2011) Up-regulation of PPAR $\gamma$, heat shock protein- 27 and -72 by naringin attenuates insulin resistance, $\beta$-cell dysfunction, hepatic steatosis and kidney damage in a rat model of type 2 diabetes. Br J Nutr 106, 1713-1723.

16. Reeves PG, Nielsen FH \& Fahey GC Jr (1993) AIN-93 purified diets for laboratory rodents: final report of the American Institute of Nutrition ad hoc writing committee on the reformulation of the AIN-76A rodent diet. $J$ Nutr 123, 1939-1951.

17. Matsuda M \& De Fronzo RA (1999) Insulin sensitivity indices obtained from oral glucose tolerance testing: comparison with the euglycemic insulin clamp. Diabetes Care 22, 1462-1470.

18. Muniyappa R, Chen H, Muzumdar RH, et al. (2009) Comparison between surrogate indexes of insulin sensitivity/resistance and hyperinsulinemic euglycemic clamp estimates in rats. Am J Physiol Endocrinol Metab 297, 1023-1029.
19. Karatepe M (2004) Simultaneous determination of ascorbic acid and free malondialdehyde in human serum by HPLC/ UV. LC GC N Am 22, 362-365.

20. Ross MH, Reith EJ \& Romrell LJ (1989) Histology. A Text and Atlas (Kimberly Kist), 2nd ed. Baltimore, MD: Williams and Wilkins.

21. Arvind K, Pradeepa R, Deepa R, et al. (2002) Diabetes and coronary artery disease. Indian J Med Res 116, $163-176$.

22. Zheng XK, Zhang L, Wang WW, et al. (2011) Anti-diabetic activity and potential mechanism of total flavonoids of Selaginella tamariscina (Beauv.) spring in rats induced by high fat diet and low dose STZ. J Ethnopharmacol 137, 662-668.

23. Sahin K, Tuzcu M, Orhan C, et al. (2011) The effects of chromium complex and level on glucose metabolism and memory acquisition in rats fed high-fat diet. Biol Trace Elem Res 143, 1018-1030.

24. Preuss HG, Echard B, Perricone NV, et al. (2008) Comparing metabolic effects of six different commercial trivalent chromium compounds. J Inorg Biochem 102, 1986-1990.

25. Jain SK, Croad JL, Velusamy T, et al. (2010) Chromium dinicocysteinate supplementation can lower blood glucose, CRP, MCP-1, ICAM-1, creatinine, apparently mediated by elevated blood vitamin $\mathrm{C}$ and adiponectin and inhibition of NFkappaB, Akt, and Glut-2 in livers of zucker diabetic fatty rats. Mol Nutr Food Res 54, 1371-1380.

26. Akude E, Zherebitskaya E, Chowdhury SK, et al. (2011) Diminished superoxide generation is associated with respiratory chain dysfunction and changes in the mitochondrial proteome of sensory neurons from diabetic rats. Diabetes 60, 288-297.

27. Derosa G \& Maffioli P (2012) Peroxisome proliferator-activated receptor- $\gamma$ (Ppar- $\gamma$ ) agonists on glycemic control, lipid profile and cardiovascular risk. Curr Mol Pharmacol 5, 272-281.

28. Benigni A, Zoja C, Campana M, et al. (2006) Beneficial effect of TGFbeta antagonism in treating diabetic nephropathy depends on when treatment is started. Nephron Exp Nephrol 104, 158-168.

29. Panchapakesan U, Chen XM \& Pollock CA (2005) Drug insight: thiazolidinediones and diabetic nephropathyrelevance to renoprotection. Nat Clin Pract Nephrol 1, 33-43.

30. Bobulescu IA (2010) Renal lipid metabolism and lipotoxicity. Curr Opin Nephrol Hypertens 19, 393-402.

31. Shen W, Hao J, Tian C, et al. (2008) A combination of nutriments improves mitochondrial biogenesis and function in skeletal muscle of type 2 diabetic Goto-Kakizaki rats. PLoS One 3, e2328.

32. Khan HB, Vinayagam KS, Moorthy BT, et al. (2012) Antiinflammatory and anti-hyperlipidemic effect of Semecarpus anacardium in a high fat diet: STZ-induced type 2 diabetic rat model. Inflammopharmacology (Epublication ahead of print version 25 January 2012).

33. Reddi A, DeAngelis B, Frank O, et al. (1988) Biotin supplementation improves glucose and insulin tolerances in genetically diabetic KK mice. Life Sci 42, 1323-1330.

34. Zhang H, Osada K, Sone H, et al. (1997) Biotin administration improves the impaired glucose tolerance of streptozocin-induced diabetic Wistar rats. J Nutr Sci Vitaminol (Tokyo) 43, 271-280.

35. Cheatham B \& Kahn CR (1995) Insulin action and the insulin signaling network. Endocr Rev 16, 117-142. 
36. Wang ZQ, Zhang XH, Russell JC, et al. (2006) Chromium picolinate enhances skeletal muscle cellular insulin signaling in vivo in obese, insulin-resistant JCR:LA-cp rats. J Nutr 136, 415-420.

37. Kuhad A \& Chopra K (2009) Tocotrienol attenuates oxidative-nitrosative stress and inflammatory cascade in experimental model of diabetic neuropathy. Neuropharmacology 57, 456-462.

38. Kuhad A, Sachdeva AK \& Chopra K (2009) Attenuation of renoinflammatory cascade in experimental model of diabetic nephropathy by sesamol. J Agric Food Chem 57, 6123-6128
39. Cheng CF, Chen HH \& Lin H (2010) Role of PPAR $\alpha$ and its agonist in renal diseases. PPAR Res 2010, 345098.

40. Tuzcu M, Sahin N, Orhan C, et al. (2011) Impact of chromium histidinate on high fat diet induced obesity in rats. Nutr Metab (Lond) 3, 8:28.

41. Zhang H, Osada K, Maebashi M, et al. (1996) A high biotin diet improves the impaired glucose tolerance of long-term spontaneously hyperglycemic rats with non-insulindependent diabetes mellitus. J Nutr Sci Vitaminol (Tokyo) 42 , 517-526. 